\title{
Researches on how to Plan the Medical Service of Hospital
}

\author{
Shi Jiahe**1, Yang Yang ${ }^{1}$, Xing Chenghao ${ }^{1}$, Jia Dongxue ${ }^{2}$ \\ ${ }^{1}$ Qiushi Honors College, Tianjin University, Tianjin, China \\ ${ }^{2}$ College of Electrical and Electronic Engineering, Shandong University of Technology, Zibo, China \\ *shijiahe_2006@126.com
}

\begin{abstract}
In recent years, the aging trend of the population is gradually increasing. Meanwhile, the residents' health awareness is also rising and medical information reform continues to deepen and China's medical service is undergoing rapid development as well. Researches on how to plan the medical service of hospital can not only avoid the waste of resources, but also effectively contribute to the development of China's medical and health services.

We solve the problem from two aspects. One is the queue rules of patients. Introduce weight innovation boldly and design a new Queuing system—combine M/M/1/00/00/PS with M/M/C/00/00/FCFS. The other is index system of evaluation which is from the perspective of both patient and hospital. Based on combining psychology and mathematics such as fuzzy math formulas, scientific and reasonable weight of indicates is obtained by Analytic Hierarchy Process Then we achieve to evaluate the model of multi-level correctly and arrange the internal resources of hospitals reasonable. Among them, combining the AHP, Delphi method and queuing theory based model to obtain the objective function by $f$ is a great innovation. In addition, taking the factors such as season and holidays, which result in movements, into consideration, related indicators are improved into a function of time subtly. And then as a basis, hospitals will get reasonable suggestion about collecting information variables. At last, hospitals achieve to make optimal use of resources within the hospital, and improve patients'satisfaction as high as possible by a full range of reforms.
\end{abstract}

Keywords: queuing theory, analytic hierarchy process, Delphi method, nonlinear programming

\section{INTRODUCTION}

As is known to all, difficult hospitalization has always been an unspeakable pain to patients and hospitals. Searching to the bottom, besides the shortage and unreasonable distribution of medical resources, the resource discordance within the hospital is also vital importance. Along with the development of the Internet and improvement of information degree, hospitals can obtain a great amount of information through modern methods. By means of medical big data, hospital are able to get fully integrated resources, as well as unified coordination, providing patients with more humanized ,personalized and effective medical service. Nevertheless, hospitals are also confronted with daze prevouiling in the age of big data, these data characterized by large amount, fine timeliness and incertitude. How to integrate a sea of info efficiently to pull together relying on the analysis and statistics of disease cure rate through big data, hospitals can render patients best therapy procedure, according to their diagnostic info. Besides, it can arrange in advance to get rid of troubles rising from conflict and waiting in a line.

Imagine 1 below diagrams a process of patient's visit to hospitalization-----general patients, after clinic diagnosis, partly leave the hospital, others receive an operation or nosocomial treatment. The latter usually need to queue at fastigium, waiting for their arrangement. There are another sort of special patients called selective operated patients, who have completed diagnosis but need elective surgery. Selective operated patients' queuing info. Classically, selective operated patients fall into 2 
categories: sick people who are in hospital a day before their operation appointment, and directly operative patients. Once the operation is performed, selective operated patients are needless to be fixed wards and queue up.

In practice, disease is often sufficient in some departments, which results in that the situation of waiting for beds is serious, and lots of patients need to line up to be in hospital. On the contrary, some departments has many empty beds, and bed occupancy rates are very low. For this reason, from the holistic perspective, hospitals can centralized the wards and beds.

At present, there is no sufficient conditions of all disease of pathological for large samples to make investigations. Abroad also imperfect in this regard. With the establishment of data systems, disease research, treatment options, and user services can be promoted simultaneously. However, hospitals can only focus on the management of health care resources in order to adapt to the arrival of the age of big data.

In order to improve the efficiency of hospital resources, please try to analyze and solve the following problems:

Problem one: information systems are not yet under fully perfect conditions, in order to meet service requirements. What data do hospitals need to collect? Please explain the reasons. Then explain how to organize and describe this information in order to improve efficiency.

Problem two: In order to improve Ward resource utilization efficiency, it's must to describe the whole process of hospitalized patients from admission to discharge correctly. But when the patients arrive at hospital and how hospital resource is distributed are uncertain. So how to reflect this uncertainty is the key to develop a deployment strategy. Try to build a mathematical model to describe the process for patients from admission to discharge.

Problem three: Please give a reasonable method of control and optimization and establish a model on overall allocation of resources of hospital ward to help improve the efficiency of hospital services and hospital resource use.

Problem four: as the seasons, holidays, and many other factors, the number of patients hospitalized is with great volatility. As a result, the hospital loads wave fiercely, which leading to the imbalance of all aspects of hospital work, seriously affecting the effectiveness of services. Please make a reasonable methods of control, in which under the condition of balancing hospital work to improve service satisfaction and efficient use of hospital beds as high as possible.

In the end, you have to provide a medical service proposal to the hospital according to your study perspective.

\section{MODEL HYPOTHESIS}

Hypothesis 1: the number of hospital workers is sufficient and once the patient is scheduled for surgery, it can be carried out on schedule.

Hypothesis 2: The patient's condition is required for surgery and hospitalization.

Hypothesis 3: Once patients enter the hospital referral, they never give up treatment nor accidental pass away.

Hypothesis 4: The influence of medical instrument is not considered.

Hypothesis 5: The data used are real and reliable.

Hypothesis 6: Patient source is unlimited, and the arrival of the patient is random.

Hypothesis 7: The impact of sudden major diseases is not considered, such as SARS, etc. 


\section{SYMBOL DESCRIPTION}

\begin{tabular}{|c|c|}
\hline $\mathrm{k}$ & The position of the patient in the team \\
\hline $\mathrm{C}_{\mathrm{n}}$ & Number of operation room / service desk \\
\hline$\lambda_{\mathrm{n}}(\mathrm{t})_{1}$ & $\begin{array}{c}\text { The proportion of the total patients in the } \\
\text { Department of the T disease }\end{array}$ \\
\hline$\mu_{\mathrm{n}}(\mathrm{t})$ & Service time for the T disease \\
\hline $\mathrm{W}_{\mathrm{t}}$ & The waiting time for one patients \\
\hline $\mathrm{L}_{\mathrm{n}}(\mathrm{t})$ & The number of beds in the Department \\
\hline $\mathrm{V}_{\mathrm{n}}$ & Operation time of the T disease \\
\hline$\eta_{\mathrm{o}}$ & Operation room utilization \\
\hline
\end{tabular}

\section{Problem Analysis}

\subsection{Background of Analysis}

1. With the development of society and the phenomenon of aging population, how to realize the reasonable allocation of medical resources is becoming a hot topic. The hospital is a complex system and the patient to the hospital time is relatively random and hard to control. What's more, State of resources when they reach the hospital is uncertainly and coordination of internal resources is difficult. Thus, the phenomenon of queuing is easy to occur. The waiting time of the hospital bed and the operation can reduce the patient's satisfaction with the hospital. However, the waiting time of the hospital bed and the operation can reduce the patient's satisfaction with the hospital. But if we blindly increase the number of the beds, the hospital bed utilization ratio will decrease. Thus, In the case of the total number of beds and the operation room, we should rationally allocate of resources to reduce patients' waiting time and improve the utilization rate of hospital bed.

2. At present, the domestic hospital managements rely on experience and general practice and they use scientific and quantitative methods, while the queuing theory in operations research is a science to solve the above problems. The queuing theory is an effective method to study the above problems quantitatively and scientifically. The hospital's medical resources mainly include ward, operation room and some other resources and the allocation of resources is determined by the management of the hospital. As for the manager of the hospital, the ratio of ward in different departments and reasonable arrangement of operation room is very essential for the integration of medical resources and the maximization of the benefits of medical institutions.

3. In this paper, the analysis of the ratio of hospital wards and operation room and the order of the patient queuing. The conclusion will be used to achieve a win-win situation of patient satisfaction and hospital resource utilization.

\subsection{Problem Analysis}

\subsubsection{Analysis of problem 1}

Queuing service system is mainly divided into three parts: input process, queuing discipline, agencies, the form is

\section{$\mathrm{A} / \mathrm{B} / \mathrm{C}$}

According to the relevant literature reports, the time of arrival of the patients to the time of $\mathrm{T}$ and the service time of the operation room are not subject to the specific distribution. As a result, we can only do the general distribution. Then, Queuing system should be recorded as $\mathrm{G} / \mathrm{G} / \mathrm{K}$, which follow the rules and characteristics of the type of the queuing system. But according to the theory of queuing 
theory, $\mathrm{G} / \mathrm{G} / \mathrm{K}$ queuing system is extremely difficult to solve. Thus, We use this set of mathematical models, $\mathrm{M} / \mathrm{M} / \mathrm{C} / \infty / \infty$ and $\mathrm{M} / \mathrm{M} / 1 / \infty / \infty$ mixed queuing system. One is a queuing system whose input process is Poisson flow, service time obeys the complex exponential distribution, and system has $\mathrm{C}$ service desk parallel services, system capacity is infinite and system source is infinite waiting for system. The other one is a queuing system whose input process is Poisson flow, service time obeys the complex exponential distribution, and system has one service desk parallel service, system capacity is infinite and system source is infinite waiting for system.

Then, we get the effective resource utilization of beds,

$$
R=\frac{\sum_{i=1}^{n}\left(Z_{i}-X_{i}+K_{i}\right)}{\sum_{i=1}^{n}\left(Z_{i}-Y_{i}\right)},
$$

Among the formula, $K_{i}$ stands for the time to observe the patient's hospitalization; $Z_{i}$ stands for the time when patient discharges. $X_{i}$ stands for the time when the patient takes the operation. $Y_{i}$ stands for the time when the patient is hospitalized.

In addition, we use the analytic hierarchy process to define the weight of different patients, and to plan the queue order of different patients. Finally, we use fuzzy psychology to define the overall level of the system.

From the perspective of queuing system, we need to know the data matrix of each disease (operation time, preoperative preparation time, postoperative recovery time, the severity of the illness, the proportion of all patients), the number of operation room, the number of various departments.

From a psychological point of view, we need to know the patient's satisfaction and the utilization rate of hospital beds for effective resources for this system, the weight of each.

\subsubsection{Analysis of problem2}

The patient entered the two queue after the diagnosis of the disease. One is the $\mathrm{M} / \mathrm{M} / 1 / \infty$ / $/$ queuing system which uses PS queuing rules. The patient's weight is made up of two parts: one is the duration when you are in the queue, and the other is the distance to do the most appropriate operation of the hospital bed. The service desk of the queuing system is the hospital bed. Whenever the other patients in the Department of the hospital discharge, there is a patient immediately added. Thus, the service of this system is 1 Units. The patient enters the ward by this queue rule, but also try to meet the hospital waiting for the operation time before entering the ward (The patient's operation time and the operation time of all patients in the cohort 2). This system represents the whole process of the patient from the clinic to the ward. The other is $\mathrm{M} / \mathrm{M} / \mathrm{C} / \infty / \infty$ queuing system which uses FCFS queuing rules. The queue is sorted according to queue 1.The service desk of the system is the operation room. Ln (the number of units in the beds) of the cohort indicates the team from admission to the preparation for the operation. There is no practical significance after Ln. This system represents a procedure for patients from outpatient to surgery.

\subsubsection{Analysis of problem 3}

The evaluation index of the system is divided into two parts: one is the satisfaction of the patients, and the other is the effective utilization of the resources of the hospital. For patient satisfaction, there are two parts; one is the length of time for the patient to be hospitalized, and the other is the duration from the hospital to the preparation of the operation. The utilization rate of hospital resources is made up of 
two parts: the utilization rate of hospital bed and the utilization rate of operation room. The bed

utilization rate is obviously determined by the formula

$$
R=\frac{\sum_{i=1}^{n}\left(Z_{i}-X_{i}+K_{i}\right)}{\sum_{i=1}^{n}\left(Z_{i}-Y_{i}\right)}
$$

mentioned previously.

According to the number of beds, it is clear that the number of beds, the shorter the patients from the diagnosis, the higher the patient satisfaction, the patient from the hospital to the preparation of the operation time, the patient satisfaction is reduced; the utilization rate of the operation room, the number of beds, the higher the utilization rate of operation room. At the same time, the number of hospital beds is a fixed value, so we need to be in accordance with the weight ratio of each department to get the best bed number, so as to get the best system.

\subsubsection{Analysis of problem4}

The incidence of different diseases, data with time changing, we can divide the function of each variable is set to $t$, in a relatively short period of time, can be regarded as a constant, with the change of time, the corresponding data is also in a constantly changing hospital can collect corresponding data, then according to the problem of three established model calculated hospital now the evaluation index and the best evaluation index. If the difference between the two is not more than $10 \%$, the number of hospital beds without change; if the difference between the two is more than $10 \%$, the hospital in accordance with the problem of three of the method to re planning the number of departments. This method not only can improve the resource utilization, but also balance the work intensity of the hospital.

\section{THE ESTABLISHMENT AND THE SOLUTION}

\subsection{The Establishment of Model1}

For patients to sort, take the patient's input process into account.

The input process is to describe how the customer according to the rules of the arrival queuing system. For customer sources, the source may be limited, and it may be infinite; for the arrival type, the customer may be a single arrival, and it may be a batch arrival; for the customers to arrive at the time, some are subject to Poisson distribution, some are subject to the K Erlang distribution, and some are equally spaced time. Poisson distribution is the most widely used distribution model, which can describe the customer arrival system from two perspectives: first, the number of customers arriving at a certain time (such as the unit time), the two is the time of the customer arrival system.

In probability theory, the probability distribution of random variable $\mathrm{N}$ is

$$
P\{N=\mathrm{n}\}=\frac{\lambda^{n}}{n !} e^{-\lambda}(\mathrm{n}=0,1, \ldots ; \lambda>0)
$$

$\mathrm{N}$ is called Poisson distribution. In the Poisson distribution, the number of customers arriving at a queuing system within the unit time is a random variable that obeys the Poisson distribution. Then, the number of customers in the queue system to reach the queuing system $N(t)$, then the probability distribution of the expression should be

$$
P\{N=\mathrm{n}\}=\frac{(\lambda t)^{n}}{n !} e^{-\lambda t}(\mathrm{n}=0,1, \ldots ; \lambda>0 ; t>0) .
$$


We called N (t) for the Poisson process, also known as Poisson flow. It satisfies the following three conditions: no aftereffect, namely the number of customers arriving in the do not want to hand over the time interval is independent of each other; stationarity, namely in $[t, t+t+\Delta t]$.

For this model, in practical terms, the patients are individually coming and are independent of each other, a certain period of time to reach number follows a Poisson distribution, arrival process is stationary (variance value is not affected by the time to reach the time interval and expected), so for the number of smooth team, we can stick weighted assignment came to an optimization of the queuing rules for the team.

First, what is the normal and reasonable queuing rules? At first, we need to consider how long the patient has been lined up. Because our model in the A queue is not the FCFS model, for a long time already lined up the team, we also need to make it ranked in the forefront of the team. Therefore, in the queue order we introduce first weight $a_{1}$, of the weight of the indicators for the patient who has ranked the long team, we will the quantification for the patient in team in position K. Relatively speaking, the patient's position weighted value $a_{1} k$ is smaller, the position of the patient in a team should be more front. In addition, we also want to consider the use of internal resources in the hospital when the patient is hospitalized. First, we assume that each department of the allocation of the operation room as a fixed value, this value should be in the Department of patients with long-term operation. Then, the utilization rate of our bed utilization rate indicates that the ward utilization rate can be determined to describe. Through simulation, we find that this function solves up very trouble, so we used Matlab simulation which can make the approximation for a polynomial function on the unit quantity. Then we give him a weight $a_{2}$. Relatively speaking, if the weighted value is smaller, in the team should be depending on the month before.

\subsection{The Establishment of Model2}

\subsubsection{Establishment of evaluation system}

For the allocation of beds, we have the following evaluation system.

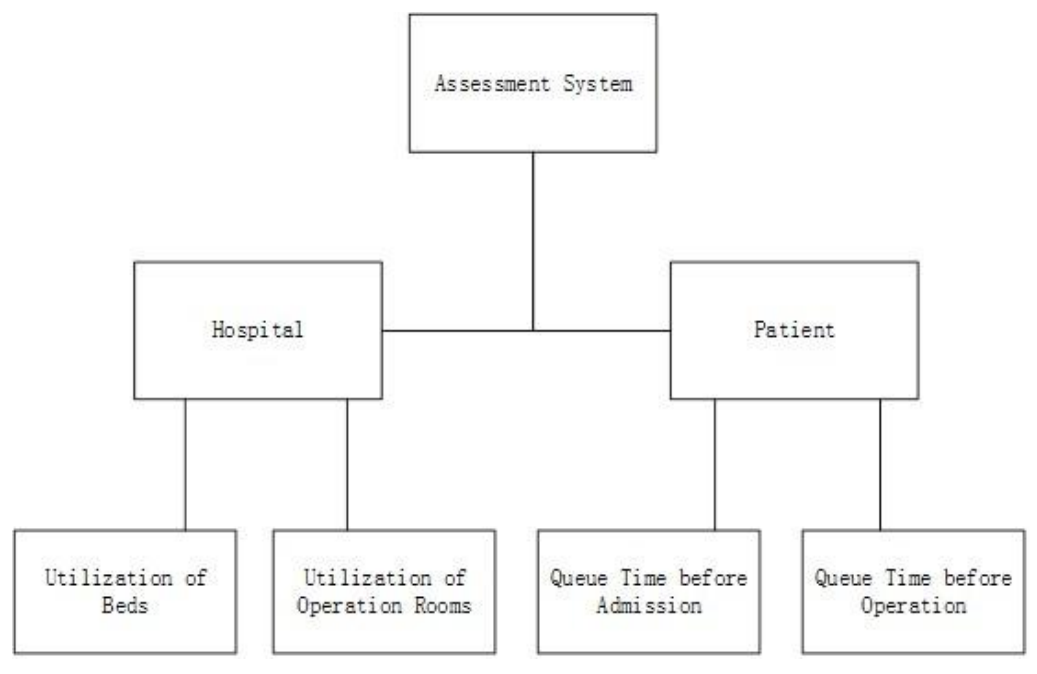

\section{Hospital angle}

Bed utilization rate, $x 1$, the higher the bed utilization is, the more reasonable the overall allocation of hospital resources are, and vice versa.

Operation room utilization rate, $\mathrm{x} 2$, the higher the utilization rate of the operation room is, the more reasonable the overall allocation of hospital resources are, and vice versa .

According to the difference of the degree of the hospital resource utilization, the weighted value of beta 1 and beta 2 was given. 


\section{Patient angle}

Waiting time for admission to the ward(patients in the outpatient service to enter the hospital waiting time), notes $\mathrm{x} 3$, waiting for the shorter the ward, the higher the patient service satisfaction, and vice versa.

Waiting time for the operation (patients admitted to the hospital after the operation of the waiting time), notes $\mathrm{x} 4$, waiting for the shorter operation time, the higher the patient service satisfaction, and vice versa.

Based on the impact of the two on the patient's service satisfaction, the size of the difference, giving different weights $\beta 3 、 \beta 4$.

In order to establish a reasonable index evaluation system, from two aspects of hospital and patients, a comprehensive evaluation index is set up.

$$
y=\beta_{1} x_{1}+\beta_{2} x_{2}+\beta_{3} x_{3}+\beta_{4} x_{4}
$$

\subsubsection{Establishment of Weighted Value}

At present, we use AHP to determine the utilization rate of the ward $\mathrm{x} 1$, operation room utilization $\mathrm{x} 2$, waiting for admission to the ward $\mathrm{x} 3$, waiting for the time $\mathrm{x} 4$ of the operation of the corresponding weight of $\beta 1 、 \beta 2 、 \beta 3 、 \beta 4$. In estimating the impact of each index on the comprehensive assessment indicators, the 2 indicators, $\mathrm{xi}$ and $\mathrm{xj}$.We use the following nine levels to quantitatively express the results of this comparison, and remember it as $\mathrm{xi} / \mathrm{xj}$.

In problem solving:

\begin{tabular}{|c|c|}
\hline The importance compared xi with $\mathrm{xj}$ & $\mathrm{xi} / \mathrm{xj}$ \\
\hline equal & 1 \\
\hline middle & 2 \\
\hline Slightly important & 3 \\
\hline middle & 4 \\
\hline important & 5 \\
\hline middle & 6 \\
\hline much more important & 7 \\
\hline middle & 8 \\
\hline far more important & 9 \\
\hline
\end{tabular}

Then we get inverse matrix

$$
\left[\begin{array}{ccccc}
1 & \frac{A_{1}}{A_{2}} & \frac{A_{1}}{A_{3}} & \ldots \ldots & \frac{A_{1}}{A_{n}} \\
\frac{A_{2}}{A_{1}} & 1 & \frac{A_{2}}{A_{3}} & \ldots \ldots & \frac{A_{2}}{A_{n}} \\
\frac{A_{3}}{A_{1}} & \frac{A_{3}}{A_{2}} & 1 & \ldots \ldots & \frac{A_{3}}{A_{n}} \\
\frac{A_{n}}{A_{1}} & \frac{A_{n}}{A_{2}} & \frac{A_{n}}{A_{3}} & \ldots \ldots & 1
\end{array}\right]
$$

We regard it as matrix $\mathrm{M}$.

According to the properties of hospital services as well as the "people-oriented", cherish life, determined to wait for stay ward time $\mathrm{x} 3$, awaiting surgery time $\mathrm{x} 4$ and ward utilization $\mathrm{x} 1$, operating 
room utilization rate $\mathrm{x} 2$, between the relative importance of; according to the psychology knowledge and hospitalization costs, etc. determine waiting for the arrival of the ward $x 3$ and wait for the relative important degree between the operation time X4. Then according to the hospital situation determine ward utilization $(\mathrm{x} 4)$ and operating room utilization between $\mathrm{x} 2$ relative important degree.

$x 1 / x 2=2 \quad x 3 / x 1=3 \quad x 3 / x 2=3 \quad x 4 / x 1=4 \quad x 4 / x 2=4 \quad x 4 / x 3=4$

Then we get matrix A:

$$
A=\left[\begin{array}{cccc}
1 & 2 & \frac{1}{3} & \frac{1}{4} \\
\frac{1}{2} & 1 & \frac{1}{3} & \frac{1}{4} \\
3 & 3 & 1 & \frac{1}{4} \\
4 & 4 & 4 & 1
\end{array}\right]
$$

The matrix A is normalized, and each column is summed, and each element of the matrix A is divided by the column's and the obtained matrix B is as follows

$$
\left[\begin{array}{c}
12.97 \% \\
9.00 \% \\
24.34 \% \\
53.69 \%
\end{array}\right]
$$

$\beta 1=12.97 \% \quad \beta 2=9.00 \% \quad \beta 3=24.34 \% \quad \beta 4=53.69 \%$

\subsubsection{Evaluation Index Formula}

Hospital angel

(1)The value of $\mathrm{x} 1$ is a constant and it equals to 1 .In another word, $\mathrm{x} 1=1$.

(2)

$$
x_{2}=\left(\frac{\sum_{j=1}^{L_{n}} V_{n}\left(t_{j}\right)}{\mu_{n}\left(t_{1}\right)}-1\right)^{2} \text {. }
$$

Patient angel

Referring to the related psychology and the theory of fuzzy psychology, patients' satisfaction is quantified by the patient waiting time, and the quantitative criteria must be seen in the appendix.

(3)The satisfaction of patients before admission

$$
\begin{gathered}
t_{1}=\sum_{i=1}^{\lambda} V_{n}\left(t_{i}\right), \\
x_{3}=e^{-\sum_{i=1}^{\lambda} v_{n}\left(t_{i}\right)^{2}} .
\end{gathered}
$$


(4)The satisfaction of patients before operation

$$
x_{4}=e^{-w^{2} t j}
$$

\section{Model Testing}

Model established above can be a good simulation of the random arrival of patient. Multiple recording and averaging y can approximately eliminate the random noises. The y changes based on the changes of Ln. The function can be obtained by fitting method, whose derivation can be obtained as well as the corresponding $\mathrm{Ln}$ of extreme value.

However, in the process of running the program, this model need to calculate the average of a large number of random numbers, so the procedure is too long. It is necessary to adopt a simplified model.

In this model, the random number of Poisson distribution is replaced by the Lambada parameter, so the step of multiple operations is eliminated. Meanwhile, the Individual in the same department is represented of the expectations of the various parameters of the Department, while, in fact, the difference between the individual is ignored. Thus, the eta parameter is constant in a certain department. So in the model, each section of the Department is the FCFS model.

Each department of the FCFS mode is set at this time when the hospital has a total of C operating table, L beds, $\mathrm{N}$ department. Vn, lambada_n, and mu_n can be obtained by statistics. The number of beds to be optimized for each of the departments of the hospital bed number Ln. They are $\mathrm{N}$ vectors.

First we determine the allocation of resources in each department of the Department (or the allocation of operations for long time) $\mathrm{Cn}$. To make rational use of the operation room, $\mathrm{Cn}$ can be expressed as a function of the operation room needs for each department. And this need can be used to characterize the patient's operating time in the unit time.

$C_{n}=C \frac{\frac{\lambda_{n}}{v_{n}}}{\sum \frac{\lambda_{i}}{v_{i}}}$

Because of this time, the research of the individual departments of the FCFS M/M/C queue, here is not to prove the reference to the theory of queuing theory. It's easy to know that

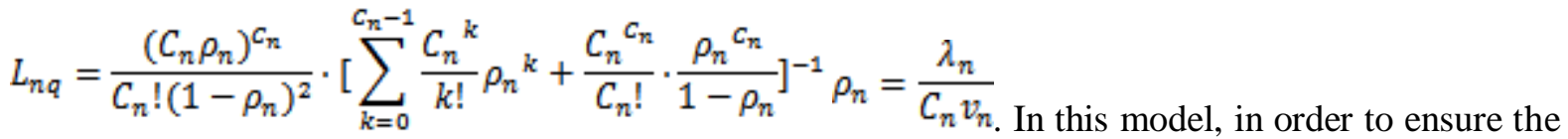
full use of the hospital bed, we deem $L_{n q}>L_{n}$.

We can know from the little formula, $W_{n q}=\frac{L_{n q}}{\lambda_{n}}$

With the same idea of calculating the utilization rate of a model of the operation room, here is the difference method $\eta_{n}=\left(\frac{L_{n}}{C_{n} v_{n}}-\frac{1}{\mu_{n}+\left(C_{n}-1\right) v_{n}}\right)^{2}$.

Wn and eta_n as the judgment index, they are normalized by the weight obtained by Delphi method, we can get a comprehensive evaluation index beta. Beta is normalized and multiplied by L to obtain an ideal solution for the Ln obtained in the current state of Lni.

$L_{n i}=\beta L$ 
Note that $\mathrm{Ln}$ is a function of Ln, Lni,, in the derivation of the Lni. So the evaluation index is introduced.

$\mathrm{Y}$ index gives the distance of the ideal combination of the $\mathrm{L} \_\mathrm{n}$ of the Department. $\mathrm{L} \_\mathrm{n}$ is the optimal solution for the $\mathrm{L} \_\mathrm{n}$ to change $\mathrm{y}$. This is the content of the nonlinear programming, which can be easily implemented using the fmincon function in matlab. Here no longer. It is indicated that the upper bound of $\mathrm{L} \_\mathrm{n}$ should be $\mathrm{L} \_n q$ in nonlinear programming due to the existence of the assumption.

$\mathrm{mu} \_\mathrm{n}=[1.62,3.58,2,1]^{\prime}$

v_n=[1.06,2.2,0.9,0.48]';

lambada_n=[1.59,3.53,1.97,0.98]';

The advantages of this model are analyzed. A total of 4 clinics, a total of 100 beds, 3 operation room.

Assuming the weight of Telfi is $[0.8,0.2]$, in this context, the matlab program can be easily obtained by using the model.

At this time the measurement

$\mathrm{y}=1.3265 \mathrm{e}-12$

Obviously, this model can give in a given weight under the condition of Delphi distribution of each department the best number of beds.

\section{The Advantage AND The Disadvantage}

\subsection{Advantages}

1. The data question 1 sought fully covers multiple indicators which impact our assessment, and these indicators can reflect the quality of patients Queuing models and bed arrangement models in many ways.

2. In question 2, the novelty of the model is reflected by using analytic hierarchy process to reinforce Queuing discipline, which make the rotations of patients more properly, and achieve to maximize team effectiveness.

3. Issue 3 introduces the related psychology and mathematics knowledge, making the distribution of beds in all departments more scientific and systematic.

4. In question 3, as the number of beds calculated may not be integers, the number of hospital beds of some illness after rounding may be less than ideal situations, in order to avoid the phenomena of shortage of bed, the system, beds shared between different diseases, is set up.

Disadvantages:

1. In the model of Question 2, it's difficult to sequence elective patients reasonably.

2. In the model of Problem 3, the impact of death caused by the failure of the operation isn't considered.

\section{MOdel REFineMENT}

1. Lambada Can be described as a variable about time, so that you can make a dynamic analysis.

2. In this model, planning on operating room $\mathrm{Cn}$ can be improved into integer programming.

3. The best solution $x$ should be an integer programming model.

4. When actually take the data into the mathematical to test the model, we can find easily that sometimes these components of the optimal solution obtained is less than the number of beds 1 . 
According to the study, as the resource available is fixed, it will product waste. In such cases beds utilization metrics should be joined.

\section{REFERENCES}

[1] Wang Kun, Li Hong. Patient satisfaction survey on application of hospital management [j]. The people's Liberation Army hospital administration journal, 2010,6:550-552

[2] Sun Honghua, Zeng Chao. Research on simulation optimization based on queuing theory in hospital [j]. Modern educational equipment, 2010,5:59-61

[3] Lu Binjie. Application of queuing theory in hospital management [j]. China digital medicine, 2006,1 (1): 41-45

[4] Wu Yuhua, Du Gang. Management Science [M].3 Edition. Tianjin: Tianjin University Press, 2009 